\title{
Maternal HIV viral load testing during pregnancy and postpartum care in Gauteng Province, South Africa
}

\author{
F Moyo, ${ }^{1,2,3}$ MSc; A H Mazanderani, ${ }^{1} \mathrm{PhD}$; T Kufa, ${ }^{1,2} \mathrm{PhD}$; G G Sherman, ${ }^{1,3,4} \mathrm{PhD}$ \\ ${ }^{1}$ Centre for HIV and STIs, National Institute for Communicable Diseases, National Health Laboratory Service, Johannesburg, South Africa \\ ${ }^{2}$ School of Public Health, Faculty of Health Sciences, University of the Witwatersrand, Johannesburg, South Africa \\ ${ }^{3}$ Paediatric HIV Diagnostics Division, Wits Health Consortium, Johannesburg, South Africa \\ ${ }^{4}$ Department of Paediatrics and Child Health, Faculty of Health Sciences, University of the Witwatersrand, Johannesburg, South Africa
}

Corresponding author: F Moyo (faithmo@nicd.ac.za)

\begin{abstract}
Background. Pregnant and breastfeeding women living with HIV (WLHIV) are a target population for elimination of mother-to-child transmission of HIV (eMTCT). However, there are limited data on maternal virological responses during pregnancy and the postpartum period in South Africa (SA).

Objectives. To review compliance of viral load (VL) testing with national guidelines and suppression rates during pregnancy and up to 9 months postpartum among WLHIV delivering in four tertiary hospitals in Gauteng Province, SA.

Methods. All women who had a point-of-care HIV VL test using Xpert HIV-1 VL (Cepheid, USA) at delivery in four tertiary obstetric units in Gauteng between June 2018 and February 2020 were included. HIV VL tests of eligible women performed up to 9 months before and after delivery were extracted from the National Health Laboratory Service's Corporate Data Warehouse. Proportions of women delivering who had antenatal and postpartum VL tests performed and their suppression rates were determined and expressed as percentages.

Results. Of 4989 eligible WLHIV (median age 31.1 years), 917 (18.4\%) had a VL performed during the antenatal period; of these, 335 (36.5\%) had a VL $\geq 50$ copies $/ \mathrm{mL}$ and 165 (18.0\%) a VL $\geq 1000$ copies/mL. At delivery, 1911 women (38.3\%) had a VL $\geq 50$ copies $/ \mathrm{mL}$ and $1028(20.6 \%)$ a VL $\geq 1000$ copies/mL. Among 627 women (12.6\%) with a VL test postpartum, 234 (37.3\%) had a VL $\geq 50$ copies/mL and $93(14.8 \%)$ a $\mathrm{VL} \geq 1000$ copies/mL. Overall, having a VL test performed during the antenatal period was associated with viral suppression at delivery and receiving a VL test postpartum $(p<0.001)$. Women with a $\mathrm{VL} \geq 50$ copies $/ \mathrm{mL}$ at delivery were more likely to be younger and to remain virally unsuppressed postpartum $(p<0.001)$ compared with women with a VL $<50$ copies $/ \mathrm{mL}$.

Conclusions. Fewer than 5\% of WLHIV with a VL at the time of delivery received VL monitoring during the antenatal and postpartum periods in accordance with national guidelines. More than $80 \%$ of WLHIV delivering had no evidence of VL monitoring during the antenatal period, and they were more likely than women who received monitoring during the antenatal period to be virally unsuppressed at delivery and to receive no VL monitoring postpartum. Women with a high VL at delivery were likely to remain virally unsuppressed postpartum. These results emphasise the need for closer monitoring of and rapid reaction to high maternal VLs during pregnancy, at delivery and postpartum for attainment of eMTCT.
\end{abstract}

S Afr Med J 2021;111(5):469-473. https://doi.org/10.7196/SAMJ.2021.v111i5.15240

Maternal viral load (VL) is closely associated with the risk of mother-to-child transmission (MTCT) of HIV. ${ }^{[1-3]}$ Frequent and timely VL testing of pregnant and breastfeeding women living with HIV (WLHIV) is therefore critical to prevent MTCT, because there is limited time for identifying women with high VLs and instituting interventions, such as switching regimens and/or intensifying adherence support, to ensure VL suppression. The South African (SA) Prevention of Mother-to-Child Transmission of HIV (PMTCT) programme has evolved to enable universal HIV testing and antiretroviral therapy (ART) coverage in the public sector. For monitoring treatment response, the PMTCT guidelines since 2015 have recommended a VL test at the first antenatal care (ANC) visit for WLHIV already on ART, irrespective of when the last VL was done. ${ }^{[4]}$ For WLHIV newly diagnosed with HIV during pregnancy, a VL is recommended after 3 months of ART use. In both groups, 6-monthly VL monitoring follows throughout pregnancy and the breastfeeding period. In November 2019, the guidelines were revised to include a VL test at delivery for all WLHIV, and the VL threshold for instituting intensified adherence support and/or regimen changes was lowered from $\geq 1000$ to $\geq 50$ copies $/ \mathrm{mL}^{[5]}$
Despite the existence of a robust PMTCT policy framework, minimal attention has been placed on maternal VL monitoring during pregnancy and the postpartum period in SA. ${ }^{[2]}$ Previous reports have found VL testing coverage as low as 30\% during pregnancy or the breastfeeding period, ${ }^{[6]}$ with frequent disengagement from care during the postpartum period, particularly among WLHIV newly initiating ART during pregnancy. ${ }^{[7,8]}$ Furthermore, $~ 20 \%$ of maternal VLs were $\geq 1000$ copies/mL during antenatal and postpartum care, ${ }^{[9]}$ while up to $37 \%$ of pregnant WLHIV have a VL $\geq 50$ copies $/ \mathrm{mL}$ at delivery in the public health sector. ${ }^{[10-12]}$ The high proportions of unsuppressed maternal VLs have important implications for vertical transmission, as elimination of mother-to-child transmission of HIV (eMTCT) requires sustained suppression of the maternal VL at $<50$ copies/mL throughout pregnancy, delivery and the breastfeeding period. The foregoing suggests that the programme is far from achieving the Joint United Nations Programme on HIV and AIDS (UNAIDS) 90-90-90 and eMTCT targets. ${ }^{[13,14]}$

SA's public health sector provides routine HIV VL testing via a well-established, centralised laboratory network that is accessible to 4300 ANC facilities nationally. ${ }^{[15]}$ However, delays exist from 
specimen collection to testing and ultimately the return of results to patients. ${ }^{[2]}$ Pointof-care (PoC) HIV VL testing has been proposed as a game changer for fast-tracking eMTCT in SA. PoC VL testing can reduce gaps along the VL monitoring cascade by accelerating the return of results to patients and facilitating prompt clinical management in cases with elevated maternal VLs. ${ }^{[16-18]}$ Previous reports suggest that PoC HIV $\mathrm{VL}$ technologies have a place in routine settings in SA. However, scale-up needs to complement existing laboratory-based systems and address site-specific health system level factors. ${ }^{[15]}$

\section{Objectives}

To review compliance of VL testing with national guidelines and suppression rates during the antenatal period and up to 9 months postpartum among WLHIV who received a PoC HIV VL test at delivery at four tertiary hospitals in Gauteng Province, SA.

\section{Methods}

Between June 2018 and February 2020, PoC HIV VL testing was piloted at four tertiary obstetric units in Gauteng - three in the City of Johannesburg and one in Tshwane District. All pregnant WLHIV presenting at labour or postnatal wards were eligible for PoC HIV VL testing using Xpert HIV-1 VL (Cepheid, USA). Specimen collection was performed by nurses and doctors as part of routine care, while testing was performed by trained PoC operators on site. Testing was performed on weekdays between $08 \mathrm{~h} 00$ and $16 \mathrm{~h} 00$. No clinical details, such as time of first HIV diagnosis, PMTCT regimen or ANC attendance, were recorded. All women who received a PoC HIV VL test at delivery were included in the study. For each PoC HIV VL that was performed, a corresponding sample was sent for routine testing to the National Health Laboratory Service (NHLS), with the two samples sharing the same laboratory barcode. Previous and subsequent HIV VL tests associated with the same patient, as per the NHLS's Corporate Data Warehouse (CDW)-generated unique patient identifier, were extracted from the NHLS CDW. HIV VL test data extracted included VLs performed up to 9 months before and after the delivery HIV VL. VLs performed up to 9 months before delivery were used as a proxy for VL monitoring during the antenatal period. Record linkage in the NHLS CDW relied on a validated probabilistic patient-linking algorithm that uses patient demographics (name, surname, date of birth, etc.) for linking test records to unique individuals. ${ }^{[19]}$

\section{Study outcomes and analysis}

The analysis evaluated proportions of women who received a VL test during the antenatal period, at delivery and within 9 months postpartum, expressed as percentages. Eligible VLs analysed included any VL performed within 9 months before and after delivery. Proportions of pregnant WLHIV with a documented VL and proportions of VL suppression during the antenatal period, at delivery and postpartum were calculated. Analyses were stratified by: (i) receipt of a PoC HIV VL result prior to discharge from hospital or not, to determine the impact of PoC HIV VL testing on the rate of retention postpartum; and (ii) maternal $\mathrm{VL} \geq 50$ copies $/ \mathrm{mL} \mathrm{v}$. $<50$ copies $/ \mathrm{mL}$ at the time of delivery. Pearson's $\chi^{2}$ test was used to test for associations between categorical variables, and Fischer's exact test in the case of sparse data. Ethics approval for the study was obtained from the Human Research Ethics Committee of the University of the

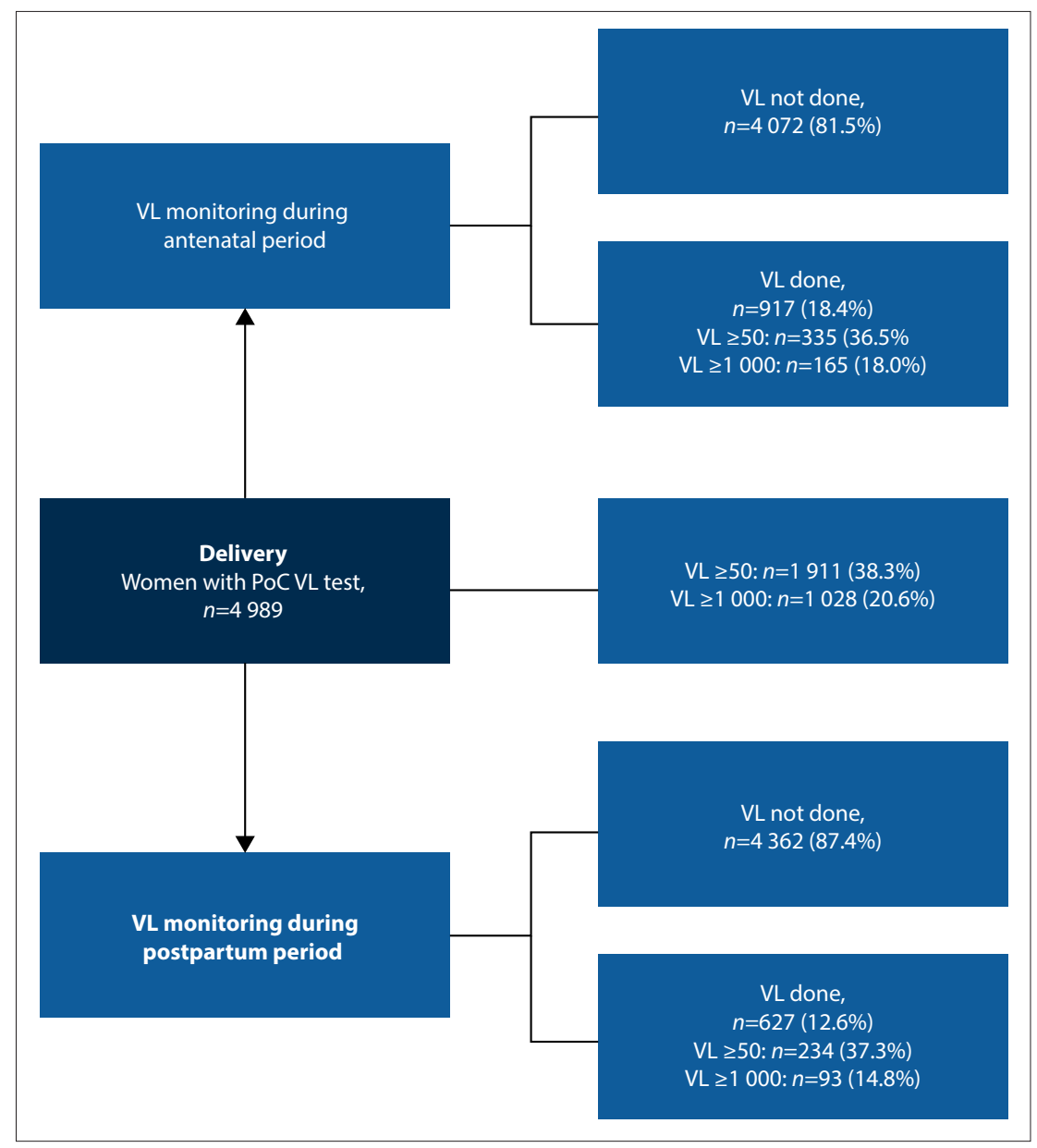

Fig. 1. VL testing compliance and rates of suppression among pregnant and postpartum women living with HIV in the cohort. $(V L=$ viral load (copies $/ m L) ; P o C=$ point-of-care. $)$

Witwatersrand (ref. no. M1711115) and the Faculty of Health Sciences Research Ethics Committee of the University of Pretoria (ref. no. 50/2018).

\section{Results}

A total of 4989 pregnant WLHIV received PoC HIV VL testing at the time of delivery from the four tertiary obstetric units between June 2018 and February 2020. Among these, 3671 women $(73.6 \%)$ received their $\mathrm{PoC}$ HIV VL result prior to discharge from the hospital and 1318 (26.4\%) did not. The median (interquartile range) maternal age was $31.1(26.6$ - 35.6) years (Table 1). A total of 917 women (18.4\%) had a documented VL during the antenatal period, of whom 335 (36.5\%) had a VL $\geq 50$ copies $/ \mathrm{mL}$ and 165 (18.0\%) a VL $\geq 1000$ copies/mL (Fig. 1). At delivery, 1911 women (38.3\%) had a $\mathrm{VL} \geq 50$ copies/mL and 1028 (20.6\%) a VL $\geq 1000$ copies $/ \mathrm{mL}$. A total of 627 women (12.6\%) had a documented VL within 9 months postpartum, of whom 234 (37.3\%) had a VL $\geq 50$ copies $/ \mathrm{mL}$ and 93 (14.8\%) a VL $\geq 1000$ copies/mL (Table 1 ). Women with a 


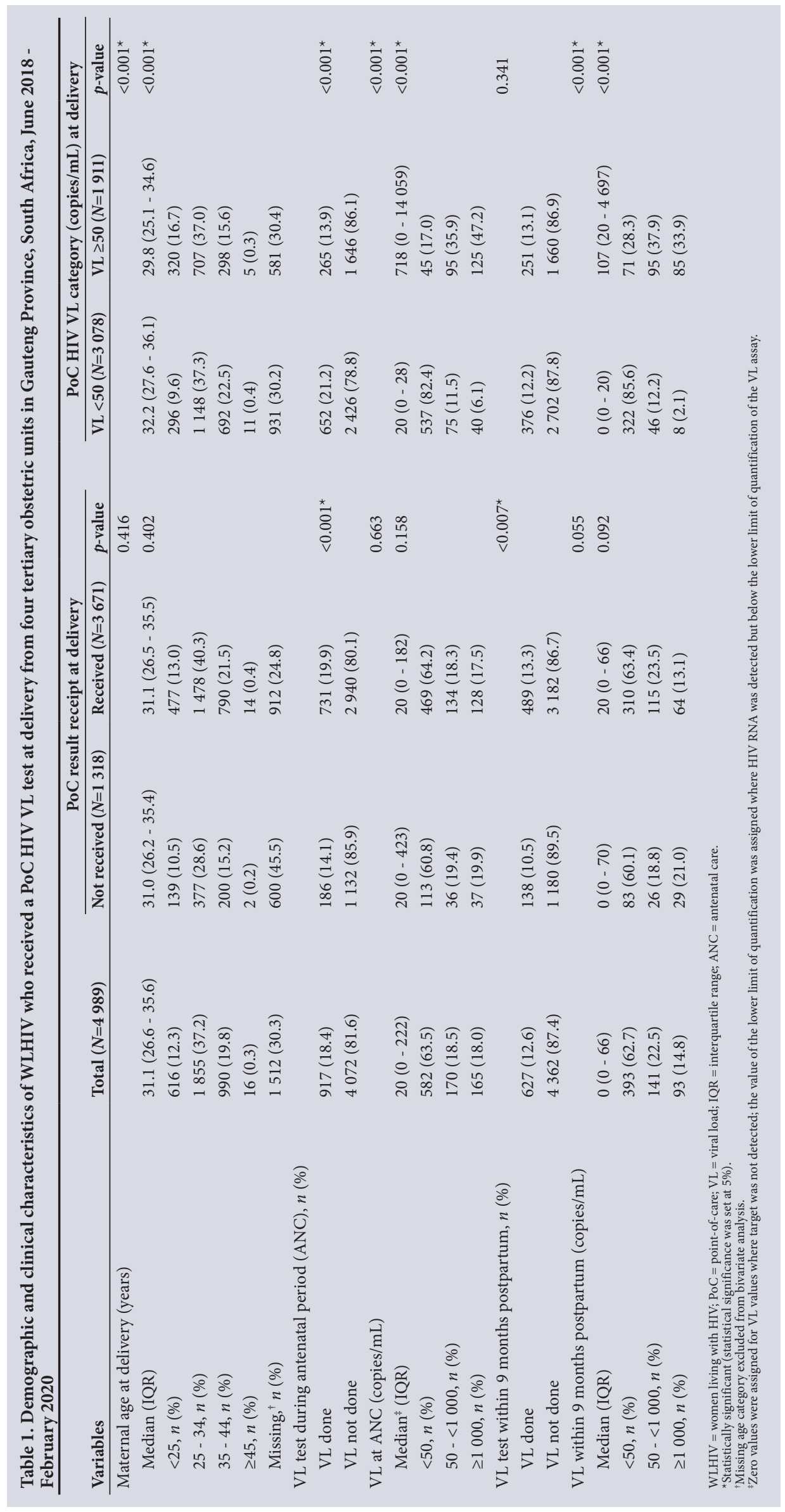

documented VL during the antenatal period $\mathrm{v}$. those without were more likely to: (i) have a $\mathrm{VL}<50$ copies $/ \mathrm{mL}$ at delivery $(n=652(71.1 \%)$ v. $n=2426$ (59.6\%); $p<0.001)$ and (ii) have a documented VL postpartum $(n=216$ $(23.6 \%)$ v. $n=411(10.1 \%) ; p<0.001)$. However, VL suppression postpartum was not associated with having a VL performed during the antenatal period $(p=0.478)$. Overall, $216(4.3 \%)$ of 4989 women received VL monitoring during the antenatal period, at delivery and within 9 months postpartum.

Receipt of a PoC VL result before discharge from the hospital was associated with having a VL test performed within 9 months postpartum. However, the association did not reach statistical significance $(p=0.055)$ (Table 1). A significant proportion of younger women (age $<25$ years) had a $\mathrm{VL} \geq 50$ copies $/ \mathrm{mL}$ at the time of delivery (Table 1). When compared with women with a VL $<50$ copies/ $\mathrm{mL}$ at the time of delivery, women with a VL $\geq 50$ copies/mL had fewer VLs during the antenatal period (265/1 911 (13.8\%) v. 652/3 078 (21.2); $p=0.001$ ) and tended to have unsuppressed VLs if they had a VL during the antenatal period (VL $\geq 50$ copies $/ \mathrm{mL}$ for $220 / 265$ women $(83.0 \%)$ v. $115 / 652(17.6 \%) ; p<0.001)$ (Fig. 1). Rates of postpartum VL monitoring were similar between the two groups at 251 (13.1\%) for women with a $\mathrm{VL} \geq 50$ copies $/ \mathrm{mL}$ at the time of delivery and $376(12.2 \%)$ for women with a VL $<50$ copies/ $\mathrm{mL}(p=0.341)$. However, women with a VL $\geq 50$ copies $/ \mathrm{mL}$ at the time of delivery were more likely to remain unsuppressed at postpartum follow-up than women with a VL $<50$ copies/mL (Fig. 1).

\section{Discussion}

We present findings from a review of compliance with national VL testing guidelines and suppression rates during the antenatal and postpartum periods in Gauteng Province. Fewer than $20 \%$ of pregnant WLHIV had evidence of VL monitoring during the antenatal period, with $37 \%$ of these women having a VL $\geq 50$ copies/ $\mathrm{mL}$ prior to delivery. At delivery, $38 \%$ and $21 \%$ of the women had a VL $\geq 50$ and $\geq 1000$ copies $/ \mathrm{mL}$, respectively. 
Overall, the rate of VL monitoring within 9 months postpartum was $13 \%$, and $37 \%$ of women with a VL test within 9 months postpartum had a VL $\geq 50$ copies $/ \mathrm{mL}$. Women with evidence of VL monitoring during the antenatal period were more likely to be virally suppressed at delivery and to receive VL monitoring during the postpartum period. We observed that a significant proportion of women aged $<25$ years had VLs $\geq 50$ copies/mL at delivery, and that women with a $\mathrm{VL} \geq 50$ copies/mL at delivery were less likely to be virally suppressed at postpartum follow-up than women with a $\mathrm{VL}<50$ copies $/ \mathrm{mL}$ at delivery. Since similar proportions of VLs $\geq 50$ copies $/ \mathrm{mL}$ were observed during the antenatal period (37\%), at delivery (38\%) and within 9 months postpartum (37\%), the findings show that a similar proportion of women are transitioning the care continuum with unsuppressed VLs. In that case, the programme is failing to identify distinct groups of women with specific needs that require targeted packages of care for maternal VL suppression. However, VL data were too few to determine the proportion of women consistently unsuppressed at all three time points.

Elimination of MTCT requires sustained maternal VL suppression from conception through pregnancy to the end of the breastfeeding period. Over $80 \%$ of women in our cohort had no evidence of VL monitoring during the antenatal period. Given the small window of opportunity for intervention between detecting a high maternal VL during the antenatal period and time of delivery, it is not surprising that $40 \%$ of these women had a VL $\geq 50$ copies $/ \mathrm{mL}$ at delivery. These findings speak to the need for improved implementation of frequent maternal VL monitoring and rapid reaction to elevated maternal VLs during pregnancy and the postpartum period. SA already has a robust policy framework that provides for optimal monitoring of maternal VLs during pregnancy. What is required are systems for improving clinical management of pregnant and postpartum WLHIV within the PMTCT programme, including PoC VL testing. ${ }^{[15]}$ PoC VL utilisation varied across our study sites, as previously reported, ${ }^{[15]}$ and these data show that approximately one in three women who received a PoC VL test at delivery did not receive their result prior to discharge from the hospital, suggesting suboptimal utilisation. Nonetheless, higher proportions of VL monitoring during the postpartum period were seen in women who received their delivery PoC HIV VL result prior to discharge from the hospital than in those who did not. Although the difference was not clinically relevant (11\% v. 13\%), the finding demonstrated the potential of PoC VL testing in improving maternal VL testing and subsequent retention in care postpartum in routine settings. This observed potential benefit of PoC VL testing is particularly important in the current context of very low maternal VL testing coverage within the national PMTCT programme, such that any intervention that improves VL testing coverage is welcome. However, further research is required to explore this association, as findings may be biased by underlying health system and patient-level factors that were beyond the scope of this study.

Evidence from various studies shows that $\sim 30-37 \%$ of women delivering in the public sector have VLs $\geq 50$ copies $/ \mathrm{mL}$ at the time of delivery. ${ }^{[9-11]}$ We present similar findings for maternal viraemia at delivery and within 9 months postpartum. These proportions are unacceptably high if the programme is in pursuit of eMTCT, especially considering recommendations for extended breastfeeding for 2 years postpartum. We found that women who had unsuppressed VLs at the time of delivery continued to be unsuppressed during the postpartum period. Even more concerning was the observed high rate of absent VL monitoring within 9 months postpartum in the cohort. This lack places HIV-exposed infants at an increased risk of MTCT during breastfeeding. ${ }^{[20-22]}$ Poor monitoring of maternal VLs during the postpartum period undermines the benefits of high ART coverage prior to delivery by compounding the risk of MTCT in the postnatal period. These findings emphasise the need for ongoing patient support and improved systems for monitoring patient care during pregnancy and the postpartum period in order to achieve eMTCT. Until clinical databases can assume this role, routine nearreal-time surveillance of maternal VLs during pregnancy, delivery and the postpartum period using the NHLS laboratory database can fast-track attainment of eMTCT and the third 90 in this population. The revised PMTCT guidelines of 2019 make provision for accurate, national surveillance of maternal VLs during pregnancy, at delivery and during the postpartum period by introducing the electronic gatekeeping (EGK) codes C\#PMTCT and C\#DELIVERY to distinguish maternal VL testing at these three time points. ${ }^{[5]}$ The EGK codes are meant to be captured on standard laboratory requisition forms for specimens submitted to and processed by the NHLS. As with other policy recommendations, the success of this initiative depends on uptake and implementation by stakeholders.

At patient level, investing in patient support services may sensitise mothers to the importance of VL monitoring and empower them to take charge of their own health and healthcare, for example young mothers and women who are struggling with adherence to treatment. The success of the PMTCT programme to date is largely attributable to implementation of broad-based and programmelevel interventions. These interventions were necessary and are still relevant. However, achieving eMTCT requires a further shift towards focusing on case management during pregnancy and the postpartum period. Poor case management may explain the constant figure of $\sim 37 \%$ of women who remain unsuppressed throughout the continuum of care in this study. These are probably women with specific needs that are not addressed by programmelevel interventions and require extra services. While this approach may be time and resource intensive, the PMTCT programme can leverage on services provided by 'MomConnect' and the community healthcare worker programme to address such needs. ${ }^{[23]}$ MomConnect is a mobile phone-based health initiative of the SA National Department of Health that sends targeted health promotion messages to pregnant women (regardless of HIV status) accessing ANC in the public health sector. The targeted health messages aim at improving both maternal and infant health. ${ }^{[23]}$ Subscription to the service is on a voluntary basis and is free of charge.

\section{Study limitations}

Interpretation of our findings requires consideration of certain limitations. We relied on the national laboratory database for VL testing patterns throughout the continuum of PMTCT care for the study cohort. However, the laboratory database is not a clinical longitudinal cohort management system. The VL testing cascade generated from laboratory data is based on availability of VL testing according to HIV monitoring guidelines. Therefore, some women may be in care but not receiving appropriate HIV testing. In addition, the laboratory database may be inaccurate for longitudinal cohort monitoring as, in the absence of a unique identifier, record linkage relies on probabilistic matching of patient demographic information. Therefore, some VL results may not have been linked to study participants as a result of inconsistent documentation of demographics (for example due to data capturing error and/or surname changes due to marriage), which would cause the patientlinking algorithm to fail to link VL results belonging to the same patient. Suboptimal linkage of VL results is particularly worth noting 
considering that pregnant women often attend multiple facilities during the antenatal, delivery and postpartum periods, ${ }^{[24]}$ and each facility may capture their personal identifiers differently. However, a manual search for longitudinal VL test results for 200 randomly selected participants was performed from the Laboratory Information System. The manual search did not yield additional VL data to results obtained from the patient-linking algorithm, suggesting reasonable performance of the linking algorithm in finding most of the HIV VLs performed on each patient and thereby validating the study findings. However, the use of markers specific for maternal VL monitoring during pregnancy and the breastfeeding period, such as the recommended EGK codes, is anticipated to provide accurate surveillance data from the laboratory database in the near future.

Another limitation is the lack of clinical information for study participants. Data on maternal ART status during the antenatal period were not available to the study to determine timing of the first VL and or the impact of ART duration on maternal viraemia during the antenatal and postpartum periods. Lastly, considering the low maternal VL testing coverage during both these periods, testing may have been biased towards unsuppressed women, which may explain why women with unsuppressed VLs during pregnancy tended to be unsuppressed during the postpartum period. Furthermore, the few VL data in this study limited a longitudinal analysis of maternal VL evolution.

\section{Conclusions}

Fewer than 5\% of WLHIV enrolled in this study received VL monitoring during the antenatal and postpartum periods according to national guidelines. Approximately one-fifth of women had evidence of VL monitoring during the antenatal period. WLHIV who did not have VL monitoring during the antenatal period were more likely to be virally unsuppressed at delivery and receive no postpartum VL monitoring. Women with a high VL at delivery were more likely to remain virally unsuppressed postpartum. These results emphasise the need for closer monitoring of and urgency in responding to elevated maternal VL during pregnancy, at delivery and postpartum for the attainment of eMTCT in SA.

Declaration. The research for this study was done in partial fulfilment of the requirements for FM's PhD in Public Health at the University of the Witwatersrand.

Acknowledgements. The authors acknowledge the NHLS CDW for providing laboratory test data.

Author contributions. GGS, FM and AHM conceived the study design and selected indicators for analysis. GGS and FM acquired the data. FM, AHM, TK and GGS analysed/interpreted the data. FM and AHM wrote the article. TK and GGS provided critical revision and final approval.

Funding. The authors acknowledge financial support for the study from the ELMA Foundation and Clinton Health Access Initiative (CHAI)/ UNITAID (project ID CHSOAFHLSTP3).

Conflicts of interest. None.
1. Mandelbrot L, Tubiana R, Le Chenadec J, et al. No perinatal HIV-1 transmission from women with effective antiretroviral therapy starting before conception. Clin Infect Dis 2015;61(11):1715-1725. effective antiretroviral therapy sta
http://doi.org/10.1093/cid/civ578

2. Myer L, Essajee S, Broyles LN, et al. Pregnant and breastfeeding women: A priority population for HIV Myer L, Essajee S, Broyles LN, et al. Pregnant and breastfeeding women: A priority population for HIV
viral load monitoring. PloS ONE 2017;14(8):e1002375. https://doi.org/10.1371/journal.pmed.1002375 viral load monitoring. PloS ONE 2017;14(8):e1002375. https://doi.org/10.1371/journal.pmed.1002375
3. Tubiana R, le Chenadec J, Rouzioux C, et al. Factors associated with mother-to-child transmission of HIV-1 despite maternal viral load $<500$ copies/ml at delivery: A case control study nested in the French perinatal cohort (EPF-ANRS CO1). Clin Infect Dis 2010;50(4):585-596. https://doi. org/10.1086/650005

4. National Department of Health, South Africa. National Consolidated Guidelines for the Prevention of Mother-to-Child Transmission of HIV (PMTCT) and the Management of HIV in Children, Adolescents and Adults. 2015. http://www.sahivsoc.org/Files/ART\%20Guidelines\%2015052015.pdf (accessed 15 July 2020).

5. Wessels I, Sherman G, Bramford L, et al. The updated South African national guidelines for the prevention of mother to child transmission of communicable infections (2019). S Afr J HIV Med 2020;21(1):a1079. https://doi.org/10.4102/sajhivmed.v21il.1079

6. Moyo F, Mazanderani AH, Bhardwaj S, et al. Near-real-time tracking of gaps in the prevention of Moyo F, Mazanderani AH, Bhardwaj S, et al. Near-real-time tracking of gaps in the prevention of
mother-to-child transmission of HIV in three districts of KwaZulu-Natal Province, South Africa. S Afr mother-to-child transmission of HIV in three districts of KwaZulu-Natal Provice

7. Phillips T, Thebus E, Bekker L-G, et al. Disengagement of HIV-positive pregnant and postpartum women from antiretroviral therapy services: A cohort study. J Int AIDS Soc 2014;17(1):19242. https:// doi.org/10.7448/IAS.17.1.19242

8. Nachega JB, Uthman O, Anderson J, et al. Adherence to antiretroviral therapy during and after pregnancy in low-income, middle-income, and high-income countries: A systematic review an metaanalysis. AIDS 2012;26(16):2039-2052. https://doi.org/10.1097/QAD.0b013e328359590f

9. Myer L, Dunning L, Lesosky M, et al. Frequency of viremic episodes in HIV-infected women initiating antiretroviral therapy during pregnancy: A cohort study. Clin Infect Dis 2017;64(4):422-427. https:// doi.org/10.1093/cid/ciw792

10. Moyo F, Haeri Mazanderani A, Murray T, et al. Characterizing viral load burden among HIVinfected women around the time of delivery: Findings from four tertiary obstetric units in Gauteng, South Africa. J Acquir Immune Defic Syndr 2020;83(4):390-396. http://doi.org/10.1097/ QAat.0000000000002267

11. Woldesenbet SA, Kufa T, Lombard C, et al. The 2017 National Antenatal Sentinel HIV Survey key findings, South Africa. National Department of Health, July 2019. https://www.researchgate.net publication/334883856_The_2017_National_Antenatal_Sentinel_HIV_Survey_Key_Findings publication/334883856_The_2017_Nation
South_Africa (accessed 16 March 2021).

12. Woldesenbet SA, Kufa T, Barron P, et al. Viral suppression and factors associated with failure to achieve viral suppression among pregnant women in South Africa. AIDS 2020;34(4):589-797. https://doi. org/10.1097/QAD.0000000000002457

13. World Health Organization. Global Guidance on Criteria and Processes for Validation: Elimination of Mother-to-Child Transmission (EMTCT) of HIV and Syphilis. Geneva: WHO, 2014. http://apps.who. int/iris/bitstream/10665/112858/1/9789241505888_eng.pdf?ua=1\&ua=1 (accessed 16 May 2020).

14. Joint United Nations Programme on HIV and AIDS (UNAIDS). 90-90-90: An ambitious treatmen target to help end the AIDS epidemic. October 2014. https://www.unaids.org/sites/default/files/ media_asset/90-90-90_en.pdf (accessed 4 February 2020).

15. Kufa T, Haeri Mazanderani A, Sherman GG, et al. Point-of-care HIV maternal viral load and early infant diagnosis testing around time of delivery at tertiary obstetric units in South Africa: A prospective study of coverage, results return and turnaround times. J Int AIDS Soc 2020;23:e25487. prospective study of coverage, results
https://doi.org/10.10.1002/jia2.25487

16. Drain PK, Dorward J, Violette LR, et al. Point-of-care HIV viral load testing combined with task shifting Drain PK, Dorward J, Violette LR, et al. Point-of-care HIV viral load testing combined with task shifting
improve treatment outcomes (STREAM): Findings from an open-label, non-inferiority, randomised improve treatment outcomes (STREAM): Findings from an open-label, non-inferiority, randomis
controlled trial. Lancet HIV 2020; 7(4):e229-e237. https:// doi.org/10.1016/s2352-3018(19)30402-3

17. ClinicalTrials.gov, US National Library of Medicine. Impact of point-of-care (POC) viral load (VL) testing on ensuring appropriate management of viremia during pregnancy to prevent vertical transmission: An observational difference-in-difference cohort study, 19th August 2019. Identifie NCT00287391. https://clinicaltrials.gov/ct2/show/study/NCT04048629 (accessed 15 May 2020).

18. Moyo S, Mohammed T, Wirth KE, et al. Point-of-care Cepheid Xpert HIV-1 viral load test in rural African communities is feasible and reliable. J Clin Microbiol 2016;54(12):3050-3055. https://doi. org/10.1128/JCM.01594-16

19. Bassett IV, Huang M, Cloete C, et al. Assessing the completeness and accuracy of South African National Laboratory CD4 and viral load data: A cross-sectional study. BMJ Open 2018:8:e021506. https://doi.org/10.1136/bmjopen-2018-021506

20. Chetty T, Newell M-L, Thorne C, et al. Viraemia before, during and after pregnancy in HIV-infected Chetty T, Newell M-L, Thorne C, et al. Viraemia before, during and after pregnancy in HIV-infected
women on antiretroviral therapy in rural KwaZulu-Natal, South Africa, 2010 - 2015. Trop Med Int women on antiretroviral therapy in rural KwaZulu-Natal,

21. Dinh T-H, Delaney KP, Goga A, et al. Impact of maternal HIV seroconversion during pregnancy on early mother to child transmission of HIV (MTCT) measured at $4-8$ weeks postpartum in South Africa 2011 - 2012: A national population-based evaluation. PloS ONE 2015;10(5):e0125525. https:// doi.org/10.1371/journal.pone.0125525

22. Patel K, Karalius B, Powis K, et al. Trends in post-partum viral load among women living with perinatal HIV infection in the USA: A prospective cohort study. Lancet HIV 2019;7(3)e184-e192. https://doi. org/10.1016/S2352-3018(19)30339-X

3. National Department of Health, South Africa. MomConnect. http://www.health.gov.za/momconnect/\# (accessed 16 March 2021)

24. Clouse K, Pettifor A, Shearer K, et al. Loss to follow-up before and after delivery among women testing HIV positive during pregnancy in Johannesburg, South Africa. Trop Med Int Health 2013;18(4):451460. http://doi.org/10.1111/tmi.12072

Accepted 3 December 2020 